\title{
STRESS SHIELDING AFTER TOTAL KNEE REPLACEMENT MAY CAUSE BONE RESORPTION IN THE DISTAL FEMUR
}

\author{
G. H. vAN LENTHE, M. C. DE WAAL MALEFIJT, R. HUISKES
}

From the University of Nijmegen, The Netherlands

Inadequate bone stock is often found in revision surgery of femoral components of total knee replacements. Our aim was to test the hypothesis that these remodelling patterns can be explained by stress shielding, and that prosthetic bonding characteristics affect maintenance of bone mass.

We made a three-dimensional finite-element model of an average male femur with a cemented femoral knee component. This model was integrated with iterative remodelling procedures. Two extreme prosthetic bonding conditions were analysed and gradual changes in bone density were calculated.

The long-term bone loss under the femoral knee component resembled clinical findings which confirms the hypothesis that stress shielding can cause distal femoral bone loss. Our study predicts, contrary to clinical findings, that an equilibrium situation is not reached after two years, but that bone resorption may continue. This hidden bone loss may be so drastic that large reconstructions are needed at the time of revision.

J Bone Joint Surg [Br] 1997;79-B:117-22.

Received 9 April 1996; Accepted after revision 15 September 1996

Recent survival analyses of total knee replacements (TKR) show that modern prostheses and cementing techniques have drastically decreased the failure rates. ${ }^{9-3}$ The Swedish National Knee Register now shows a cumulative revision rate of about $8 \%$ at ten years ${ }^{3}$, the main reason being aseptic loosening (46\%). At revision of femoral components, inadequate bone stock is often apparent ${ }^{4-6}$ and radiological studies have also shown distal femoral

G. H. van Lenthe, MSc,

$\mathrm{R}$. Huiskes, $\mathrm{PhD}$, Professor

Biomechanics Section

M. C. de Waal Malefijt, MD, PhD,

Institute of Orthopaedics, University of Nijmegen, PO Box 9101, 6500

HB Nijmegen, The Netherlands.

Correspondence should be sent to Professor R. Huiskes.

(C)1997 British Editorial Society of Bone and Joint Surgery 0301-620X/97/16808 \$2.00 bone resorption, particularly behind the anterior flange. ${ }^{7,8}$ Animal experiments have also indicated that considerable bone remodelling may take place in the distal femur. ${ }^{9}$

Petersen et $\mathrm{al}^{10}$ measured postoperative bone mineral density (BMD) in the distal femur, using dual-energy X-ray absorptiometry (DEXA); they found an average decrease of $36 \%$ in BMD in the anterior distal femur after two years. The exact amount of distal femoral bone loss is uncertain, because radio-opaque pegs or intercondylar parts of the femoral component shield much of the distal femur from radiography or DEXA measurements.

It is generally accepted that stress shielding of the proximal femur is the cause of bone loss after total hip replacement. A reduction in mechanical stresses was also found in the distal femur due to placement of a knee prosthesis; these reductions were found using finite element models. ${ }^{11-13}$ From studies of THR it is known that bonding characteristics also influence the patterns of bone resorption around hip stems.

Our aim was to determine whether the remodelling patterns in the distal femur can be explained by stress shielding, and whether prosthetic bonding characteristics affect maintenance of bone mass. We have therefore investigated the relationship between mechanical loading and the resorption of periprosthetic bone, using computer-simulation models, based on finite-element (FE) analysis and strain-adaptive bone remodelling theory.

\section{METHODS}

Finite-element model. From a collection of 250 femora, we selected ten bones with similar dimensions in the distal area, to represent average values for a normal man. ${ }^{14-17}$ Quantitative CT was performed at three specified positions in the distal femur, and used to calculate bone mineral content, average BMD, moments of inertia and average Young's moduli. These values were compared for the ten femora and the average bone was selected for full investigation.

The geometry of this femur was determined from 28 cross-sectional CT scans, separated by $4 \mathrm{~mm}$ distally, to a maximum of $16 \mathrm{~mm}$ more proximally, to cover a total length of $232 \mathrm{~mm}$. These scans were then transferred to a graphics computer programme, and used to construct a 

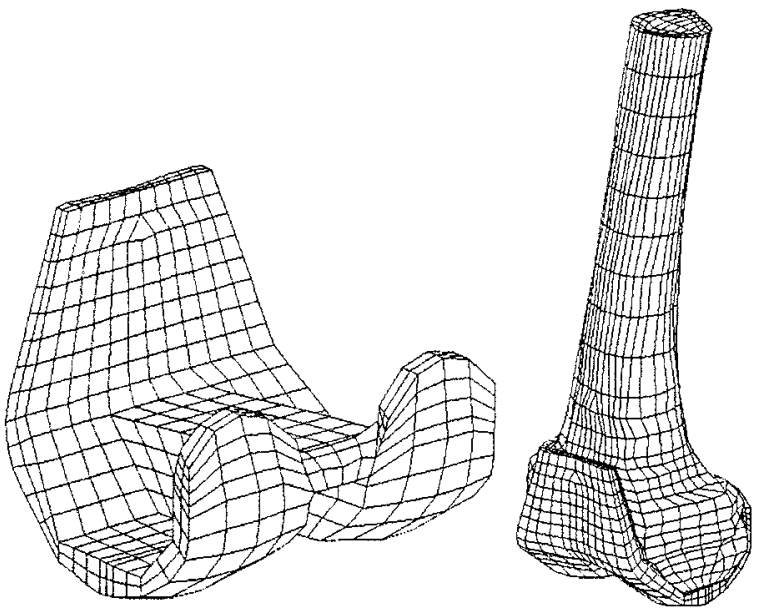

Fig. 1

FE model of the prosthesis and the operated femur.

three-dimensional FE model which consisted of 2520 8-node isoparametric brick elements.

We made an FE model of the femoral component of a total knee prosthesis (PFC with intercondylar box, Johnson \& Johnson, Bracknell, UK) and connected this to the FE model of the intact femur. Elements representing the cement layer were added. The postoperative femur model, including prosthesis and bone cement, consisted of 3116 8-node isoparametric brick elements, 385 gap elements and 4650 nodes (Fig. 1). The gap elements were positioned between the prosthesis and the cement to allow relative movement between them, but keeping the two surfaces from penetrating each other. Two kinds of analysis were performed: one with a fully unbonded interface, using the gap elements without friction, and one with a fully bonded interface, at which no micromovement was allowed.

The prosthetic Co-Cr alloy and the bone cement (PMMA) were assumed to have linear elastic, homogenous and isotropic properties, with Young's moduli of $210 \mathrm{GPa}$ and $2.1 \mathrm{GPa}$, and Poisson's ratios of 0.3 and 0.4 , respectively. The Young's moduli of the bone elements were derived from their apparent densities, calculated from the CT-density values. Hvid et $\mathrm{al}^{18}$ found a very high linear correlation between CT value and apparent density. The maximum apparent density in the FE model was normalised at $1.73 \mathrm{~g} / \mathrm{cm}^{3}$. The Young's modulus (E) of each bone element was determined from the actual apparent density $(\rho)$ according to Carter and Hayes ${ }^{19}$ as follows:

$$
\mathrm{E}=3790 \rho^{3} \mathrm{Mpa} \text {. }
$$

Poisson's ratio for all bone elements was assumed to be 0.3 .

Knee loading. We applied three load cases out of a normal daily loading cycle. The knee forces in the first and second case represented those during fast level walking at $1.7 \mathrm{~m} / \mathrm{s}$ at $15 \%$ and $45 \%$ of the walking cycle, one for the peak forces just after heel strike and one for the peak forces before toe-off. These data were obtained from three-dimensional gait analysis. ${ }^{20,21}$ The third load case represented the peak forces during cycling. ${ }^{22}$ By creating a statically determinate three-dimensional knee model, these forces and moments of force acting on the knee were calculated to forces working at the medial and lateral condyles of the tibiofemoral joint and at the patellofemoral joint (similar to those of Morrison). ${ }^{23}$ Contact points between the tibia and the femur, required for these calculations, were estimated roughly from the model of Yamaguchi and Zajac ${ }^{24}$; contact points for the patellofemoral joint were estimated from that of Van Eijden et al. ${ }^{25}$ An overview of the estimated tibiofemoral and patellofemoral contact forces is given in Table I. $F_{c c}$ is the force along the caudal-cranial axis, defined from a point between the femoral condyles towards the centre of the femoral head. $F_{m l}$ is the force along the mediolateral axis, defined as perpendicular to the caudalcranial axis and directed laterally. $\mathrm{F}_{\mathrm{ap}}$ is the force along the anteroposterior axis, which is perpendicular to both previously defined axes and is directed posteriorly.

Remodelling simulation. Long-term prediction of apparent bone density was based on a strain-adaptive bone remodelling theory ${ }^{26-29}$ used in conjunction with the FE code (MARC Analysis Corporation, Palo Alto, California). In this theory, bone cells are assumed to react to local deviations in strain energy per unit of mass. In each time step of the simulation, the remodelling signal $S$ is compared with the reference, preoperative, signal $S_{\text {ref }}$ in the corresponding location. If $\mathrm{S}-\mathrm{S}_{\mathrm{ref}}$ is positive then bone is formed, and if it is negative bone is lost. A 'dead zone' was assumed with a threshold level of $\mathrm{s}=0.75 \mathrm{~S}$. Below this level, no bone loss or bone formation was assumed to take place. ${ }^{26}$ The use of this threshold level was known to produce realistic results in simulations of non-cemented hip replacements in man. $^{27,30,31}$ Our simulations continued until the treated

Table I. Overview of the three different load cases applied to the FE model (see text)

\begin{tabular}{|c|c|c|c|c|c|c|c|c|c|}
\hline \multirow{3}{*}{$\begin{array}{l}\text { Load } \\
\text { case }\end{array}$} & \multicolumn{6}{|c|}{ Tibiofemoral contact } & & & \\
\hline & \multicolumn{3}{|c|}{ Medial condyle } & \multicolumn{3}{|c|}{ Lateral condyle } & \multicolumn{3}{|c|}{ Patellofemoral contact } \\
\hline & $\mathbf{F}_{\mathrm{ml}}(\mathbf{N})$ & $\mathbf{F}_{\text {ap }}(\mathbf{N})$ & $F_{\text {cc }}(\mathbf{N})$ & $\mathbf{F}_{\mathrm{ml}}(\mathrm{N})$ & $\mathbf{F}_{\text {ap }}(\mathbf{N})$ & $\mathbf{F}_{\text {cc }}(\mathbf{N})$ & $\mathbf{F}_{\mathrm{ml}}(\mathbf{N})$ & $\mathbf{F}_{\text {ap }}(\mathbf{N})$ & $\mathbf{F}_{\text {cc }}(\mathbf{N})$ \\
\hline 1 & -23.0 & -932.5 & 1362.0 & -11.0 & -734.5 & 664.5 & 339.0 & 1546.0 & 754.0 \\
\hline 2 & -24.0 & 7.5 & 714.0 & -8.0 & 23.0 & 238.0 & 27.0 & 94.0 & 13.0 \\
\hline 3 & -37.5 & -320.5 & 152.5 & -12.5 & -222.0 & 51.0 & 121.5 & 352.5 & 610.5 \\
\hline
\end{tabular}


bone was fully adapted to the mechanical changes produced by the implant. The mathematical formulation of the remodelling process is available from the corresponding author $(\mathrm{RH})$ on request.

Calculated computer time is related to real time by a time constant $\tau$ which was determined empirically by comparing the results of two-year animal experiments and associated computer simulations. ${ }^{29}$ This showed that $\tau$ is about $130 \mathrm{~g}^{2} \mathrm{~mm}^{-2} \mathrm{~J}^{-1} \mathrm{month}^{-1}$. As human metabolism is slower than that in the dogs used in the animal experiment, it is to be expected that bone turnover will be slower, hence $\tau$ will be lower in man. For this study, we assumed arbitrarily that $\tau_{\text {dogs }}=4 \tau_{\text {man }}$, implying that bone turnover in man is four times slower than in dogs.

To be able to visualise the postoperative changes in bone density, and to compare them with clinical results, we developed a DEXA simulation program which could produce projections of the BMD patterns which would be found if the FE model were a real bone. The DEXA simulation algorithm superimposes a large voxel set on the FE model of the bone. A calculation is then made to determine the element in which the midpoint of each voxel is positioned. The density at the midpoint of the voxel is interpolated from the density values in the nodes of the determined element and the amount of bone in each voxel is calculated. These voxel data are then superimposed in the frontal and lateral planes to provide two two-dimensional projections, which resemble DEXA scans.

\section{RESULTS}

Bone resorption in the distal femur was quantified by calculating the time-dependent BMD changes in five regions of interest (ROI). When these five ROIs had reached remodelling equilibrium they showed a wide range of BMD changes from a local BMD increase of $3 \%$ to a decrease of $96 \%$ (Fig. 2). Bone loss occurred particularly at the most distal part of the femur. For both the bonded and unbonded simulations the two most distal ROIs showed the largest and fastest decrease in BMD, with more and faster bone loss in the bonded example. In the bonded case, most loss was predicted in the anterior distal ROI (No. 1); in the unbonded case it was in the posterior distal ROI (No. 5). The long-term decreases in BMD in these two distal ROIs were $96 \%$ and $91 \%$ in the bonded simulation and $62 \%$ and $68 \%$ in the unbonded simulation. In the distal anterior femur, bone loss was greater in the lateral than in the medial condyle (Fig. 3a). These simulated DEXA scans show BMD values immediately after operation and when equilibrium had been reached. The prosthesis would have obscured the bone, and it was therefore removed from the FE model before the DEXA scans were made, as was the cement layer. In the bonded case, the lateral condyle was almost completely resorbed; in the unbonded case, bone loss was somewhat less.

Bone resorption in the bonded simulation was not only more extensive, but also faster, than in the unbonded simulation. BMD decreases in the two most distal ROIs were in the bonded simulation almost equally fast, taking an estimated 18 months before $25 \%$ of the bone in the condyles had been resorbed, 3.5 years for $50 \%$ loss and 8 years for $75 \%$ loss. In the unbonded simulation, bone loss in ROI 5 was much faster than in ROI 1. For ROI 5 there was $25 \%$ and $50 \%$ bone resorption at 1.75 and 5 years, respectively. In ROI 1, these periods were 5 and 28 years, respectively.

Some bone formation was also predicted. In both the bonded and unbonded simulations an area of BMD increase was found, which started at the proximal part of the anterior flange of the prosthesis (Fig. 4). In the bonded simulation, the increase in BMD started at the proximal tip of the prosthesis. The area behind the flange was stress shielded, as can be concluded from the $49 \%$ decrease in BMD in ROI 2. In the unbonded case, a large area of bone densification began somewhat more distally, with a longterm increase in BMD of $3 \%$ in ROI 2. There was another area of bone densification at the most proximal edge of the posterior part of the condyles; this was predicted only in the

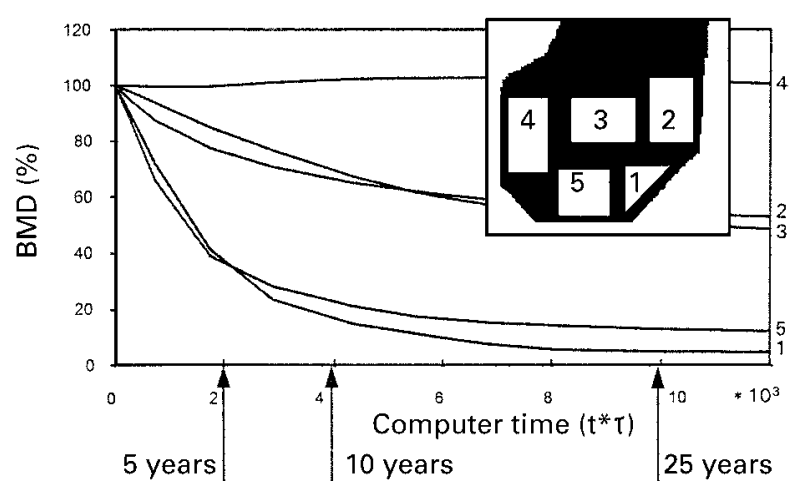

Fig. 2a

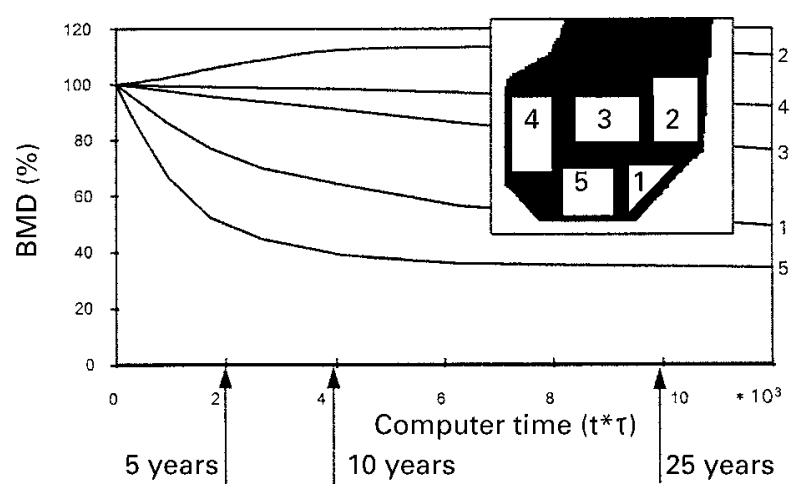

Fig. 2b

Time-dependent changes in BMD in bonded (a) and unbonded (b) analysis. The BMD value directly after operation is set to $100 \%$. 


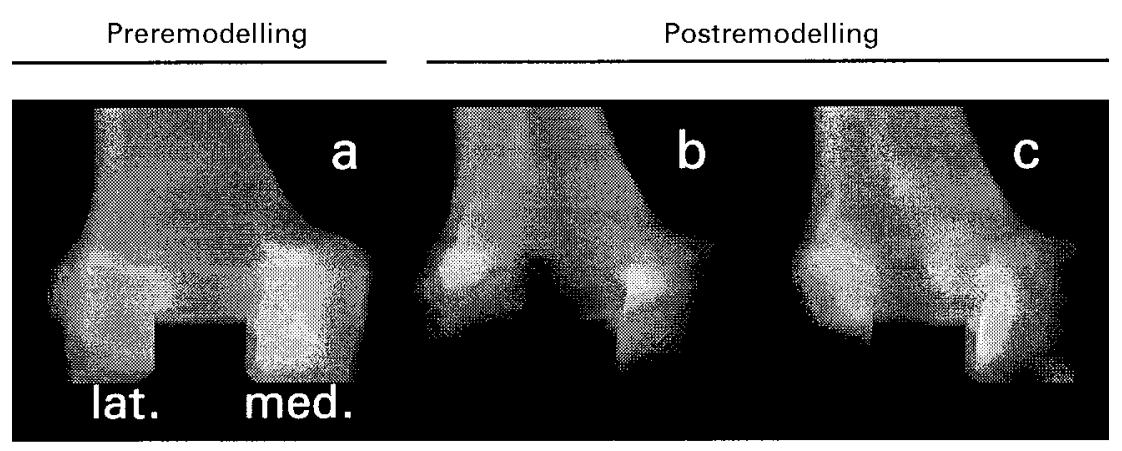

Bonded

Unbonded

Preremodelling Postremodelling

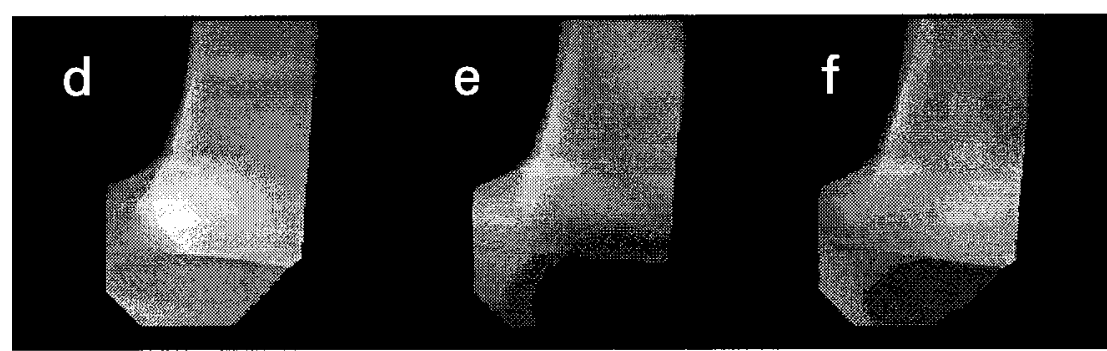

Bonded

Unbonded
Fig. 3

Simulated frontal (a-c) and lateral (d-f) DEXA scans. The prosthesis and the cement were removed from the FE model before the DEXA simulation was carried out.

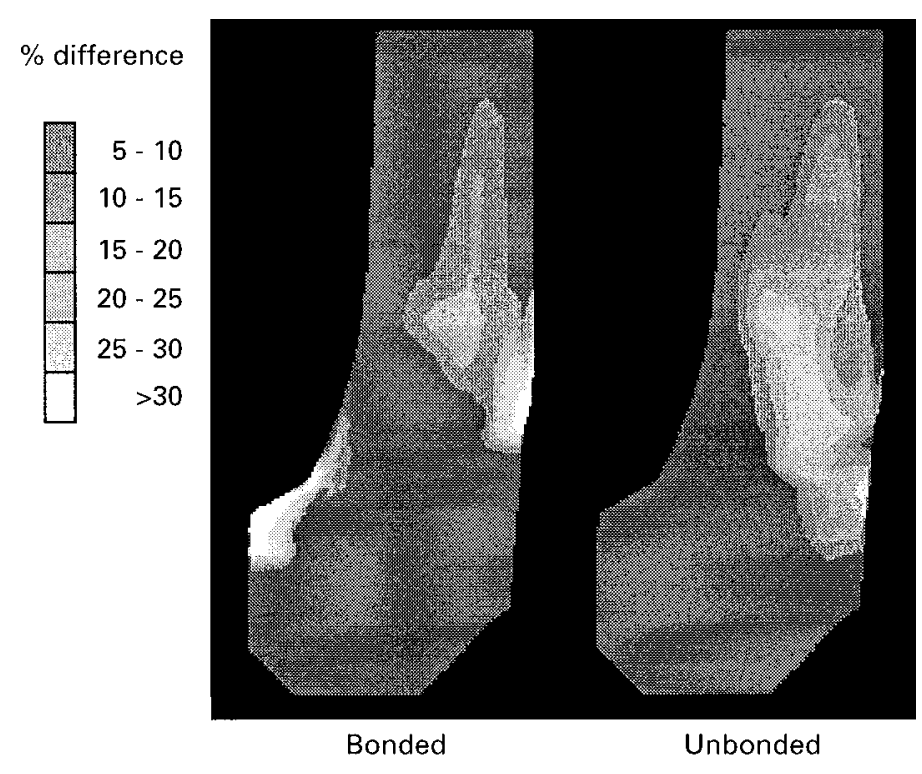

Fig. 4

Long-term increases in BMD in bonded and unbonded analysis calculated from lateral DEXA scans and expressed as a percentage change compared with directly postoperative BMD.

bonded simulation. Distal to this there was some bone loss which resulted in a net $9 \%$ loss of BMD in ROI 4 . The unbonded simulation showed bone loss in ROI 4 both proximally and distally, to an average of $27 \%$.

\section{DISCUSSION}

Our study aimed to determine whether patterns of bone resorption under femoral TKR components could be explained by strain-adaptive bone remodelling. We therefore constructed a three-dimensional FE model of a distal femur with a total knee prosthesis. We used this in conjunction with an iterative remodelling procedure designed to predict the time-dependent changes in bone mass. Similar simulations of periprosthetic bone remodelling after THR have produced realistic results, as compared with those of animal experiments ${ }^{28,29}$ and clinical retrieval studies. ${ }^{31}$ It must be made clear that our study was exploratory in 
nature, and not necessarily very precise. Several major assumptions and simplifications were used for the simulation model and no explicit validation was possible.

We used loading conditions which we calculated from analyses of fast walking and seated cycling. The external forces and moments of force from these two studies were used in conjunction with anatomical data for the knee to calculate the forces at the different joint surfaces. We realise that daily activity consists of more than walking and cycling and that in real life, the loads on joints are highly variable. The use of the site-specific remodelling theory, however, in which loads are assumed to be equal before and after the implantation make the precise loading conditions of less importance. ${ }^{26} \mathrm{We}$ assumed that the three loading conditions adequately represented normal daily activity for our purpose.

At the unbonded interface, we assumed that there was no friction. This is a simplification, but the fluids in the knee do provide some lubrication at this interface, and friction will therefore probably be low.

The dead-zone threshold level below which no remodelling will occur is known to have an important influence on the amount of bone eventually lost or gained, and also to influence the rate of remodelling. We used a threshold level of $\mathrm{s}=0.75 \mathrm{~S}$, because it had produced realistic results in simulations of non-cemented clinical hip replacements. $^{27,31}$

Biochemical or biomechanical reactions other than energy-dependent bone remodelling were not taken into account. For example, it is likely that the infiltration of wear debris causes histiocytic responses. ${ }^{32}$ This debris may gain access to the distal femoral metaphysis through the bone-implant interface, and we took no account of such reactions.

Another factor which will influence the result is the initial distribution of bone density. The postmortem analysis of 11 well-functioning unilateral hip replacements showed that periprosthetic bone loss was highly correlated with the mineral content of the contralateral hip. ${ }^{33}$ Bone loss was greater in femora with initially low bone mineral content than in those with a high bone mineral content. This phenomenon was also seen in our remodelling simulations of THR. ${ }^{27}$ The aim of our present study was explanatory rather than precise and we have therefore not yet studied this variable but it is clear that the actual amount of bone resorption will vary considerably in a population of patients.

At present the only possible validation of our findings is from published reports of quantitative radiological data. In a DEXA study, Petersen et $\mathrm{al}^{10}$ found an average $36 \%$ decrease in BMD in the anterior distal femur after two years. Cameron and Cameron ${ }^{7}$ and Mintzer et $\mathrm{al}^{8}$, evaluating different prostheses with conventional radiographs, found bone loss in the anterior distal femur within three months to one year in about $70 \%$ of all TKR cases reviewed. To compare these results with the predicted
BMD values in our study, we calculated the time by which the BMD in the distal femur had decreased by $30 \%$. We chose this level because a BMD decrease of $30 \%$ is needed before the loss becomes visible on conventional radiographs. ${ }^{34}$ For the bonded analysis $30 \%$ losses in ROI 1 and ROI 5 were reached after 23 and 19 months, respectively. In the unbonded analysis this level of loss was reached after 25 months in ROI 5, and only after 80 months in ROI 1. These results are similar to those of Petersen et $\mathrm{al}^{10}$ for the anterior distal femur, although our results are only rough estimates because the value of the time constant $\tau$ was chosen arbitrarily. The prediction of the correct time scale is difficult because little quantitative clinical data are available for the estimation of time constants for the remodelling equations.

In the other three ROIs (2, 3 and 4) bone resorption was much less. In the bonded analysis, $30 \%$ loss took 7.5 to 10 years. In the unbonded analysis only ROI 3 eventually reached a $30 \%$ BMD reduction. These results, combined with the common finding that at revision a cemented TKR is at least partially unbonded, may explain why radiological bone resorption in the distal femur is hardly ever found after placement of a prosthesis with an intercondylar box. When revision surgery is needed for any reason, however, only then is it found that the distal bone quality is much reduced, requiring major reconstruction.

Our predictions (Fig. 3a) showed that for both the bonded and unbonded cases, the BMD dropped more on the lateral than on the medial side. This can be explained by considering the position of the anterior flange of the prosthesis in relation to the shaft. Proximally, the flange is more medial in relation to the bone, with its proximal end sticking out somewhat more medially. This produced a difference between transfer of the forces applied at the medial and lateral condyles. We calculated that the interface forces at the distal lateral condyle were reduced relatively more than those at the distal medial condyle, implying more stress shielding and more bone loss.

Our results predicted some important effects of the bonding conditions of the implant. Remodelling studies of THRs have also shown clearly that interface conditions may have a large influence on bone resorption ${ }^{28,35,36}$ and on interface stresses. ${ }^{36}$ In our study, we examined the influence of two bonding conditions on bone resorption, fully bonded and fully unbonded. These can be seen as two extreme conditions for changes in BMD after TKR. We have shown that bone loss in the distal femur is more severe and occurs faster in the bonded than in the unbonded simulation, and this can be related to the extent of stress shielding. In the bonded case, knee forces were transferred to the bone more proximally as shown by the bone densification near the anterior and posterior flanges and by the severe loss of bone distally. In the unbonded case, these forces were shifted anteriorly, reducing the stresses in the posterior and distal regions. This initial stress shielding effect has already been demonstrated. ${ }^{11-13}$ We showed that it continues during 
remodelling. In the bonded simulation, a large part of the load was transferred as shear stresses through the proximal anterior flange. In the unbonded case, the intercondylar box carried a much greater part of the load and forces near the anterior flange were increased, leading to local bone formation.

Conclusions. Our results indicate that the remodelling simulation does represent an actual remodelling process. The bonding characteristics of the implant had significant effects on the long-term conservation of BMD. As compared with the bonded configuration, BMD values in the unbonded distal femur were higher and the rate of bone loss was lower. The predicted long-term bone loss resembled the actual clinical findings up to a period of two years and can therefore be explained as a long-term effect of stress shielding. Contrary to estimates from radiographs, our study predicts that equilibrium is not reached after two years, but that bone resorption continues for much longer. This hidden loss in bone stock, with almost total resorption of the distal condyles in the equilibrium situation, may be so drastic that at the time of revision some complex reconstructions are necessary.

Our results suggest that component design and the unbonding of the prosthesis may reduce the amount of stress shielding in the distal femur and lead to less bone resorption.

This research project was sponsored in part by the 'Dutch Alternatives to Animal Experiments Platform' and by Johnson \& Johnson Orthopaedics R\&D.

Although none of the authors have received or will receive benefits for personal or professional use from a commercial party related directly or indirectly to the subject of this article, benefits have been or will be received but are directed solely to a research fund, foundation, educational institution, or other non-profit institution with which one or more of the authors is associated.

\section{REFERENCES}

1. Rand JA, Ilstrup DM. Survivorship analysis of total knee arthroplasty. Cumulative rates of survival of 9200 total knee arthroplasties. J Bone Joint Surg [Am] 1991;73-A:397-409.

2. Ranawat CS, Flynn WF, Saddler S, Hansraj KK, Maynard MJ. Long term results of the total condylar knee arthroplasty: a 15-year survivorship study. Clin Orthop 1993;286:94-102.

3. Knutson K, Lewold S, Robertsson O, Lidgren L. The Swedish knee arthroplasty register: a nationwide study of 30,003 knees 1976-1992. Acta Orthop Scand 1994;65:375-86.

4. Rand JA. Revision total knee arthroplasty using the total condylar III prosthesis. J Arthroplasty 1991;6:279-84.

5. Whiteside LA. Cementless revision total knee arthroplasty. Clin Orthop 1993;286:160-7.

6. Tsahakis PJ, Beaver WB, Brick GW. Technique and results of allograft reconstruction in revision total knee arthroplasty. Clin Orthop 1994;303:86-94.

7. Cameron HU, Cameron G. Stress-relief osteoporosis of the anterior femoral condyles in total knee replacement. A study of 185 patients. Orthop Rev 1987;16:449-56.

8. Mintzer CM, Robertson DD, Rackemann S, et al. Bone loss in the distal anterior femur after total knee arthroplasty. Clin Orthop 1990;260:135-43.

9. Bobyn JD, Cameron HU, Abdulla D, et al. Biologic fixation and bone modeling with an unconstrained canine total knee prosthesis. Clin Orthop 1982;166:301-12.

10. Petersen MM, Olsen C, Lauritzen JB, Lund B. Changes in bone mineral density of the distal femur following uncemented total knee arthroplasty. J Arthroplasty 1995;10:7-11.
11. Walker PS, Granholm J, Lowrey R. The fixation of femoral components of condylar knee prostheses. Eng Med 1982;11:135-40.

12. Angelides M, Chan K, Ahmed AM, Joly L. Effect of total knee arthroplasty on distal femur stresses. Trans Orthop Res Soc 1988;13:475.

13. Tissakht M, Chan K, Ahmed AM. Bone remodeling in the distal femur with TKR: correlation with mechanical parameters. Trans Orthop Res Soc 1992;17:321.

14. Seedhom BB, Longton EB, Wright V, Dowson D. Dimensions of the knee: radiographic and autopsy study of sizes required by a knee prosthesis. Ann Rheum Dis 1972;31:54-8.

15. Erkman MJ, Walker PS. A study of knee geometry applied to the design of condylar prostheses. Biomed Eng 1974;9:14-7.

16. Mensch JS, Amstutz HC. Knee morphology as a guide to knee replacement. Clin Orthop 1975;112:231-41.

17. Mizrahi J, Benaim E. Minimization of the gliding index: criterion for the generation of the surfaces of a knee endoprosthesis. J Biomech 1987;20:851-62.

18. Hvid I, Bentzen SM, Linde F, et al. X-ray quantitative computed tomography: the relations to physical properties of proximal tibial trabecular bone specimens. J Biomech 1989;22:837-44.

19. Carter DR, Hayes WC. The compressive behaviour of bone as a twophase porous structure. J Bone Joint Surg [Am] 1977;59-A:954-62.

20. Koopman HFJM. The three-dimensional analysis and prediction of human walking. PhD thesis, University of Twente, FEBO, Enschede, The Netherlands, 1989.

21. Van Lenthe GH. Gait analysis of walking with walking aids. M.Sc thesis (in Dutch), original title: 'Gangbeeldanalyse van het lopen met loophulpmiddelen', Dep. Of Mech Eng, report no. BW-50, University of Twente, Enschede, The Netherlands, 1994.

22. Ruby P, Hull ML, Hawkins D. Three dimensional knee joint loading during seated cycling. J Biomech 1992;25:41-53.

23. Morrison JB. The mechanics of the knee joint in relation to normal walking. J Biomech 1970;3:51-61.

24. Yamaguchi GT, Zajac FE. A planar model of the knee joint to characterize the knee extensor mechanism. J Biomech 1989;22:1-10.

25. Van Eijden TM, Kouwenhoven E, Verburg J, Weijs WA. A mathematical model of the patellofemoral joint. J Biomech 1986;19:219-29.

26. Huiskes R, Weinans H, Grootenboer HJ, et al. Adaptive boneremodeling theory applied to prosthetic-design analysis. J Biomech 1987;20:1135-50.

27. Huiskes R, Weinans H, van Rietbergen B. The relationship between stress shielding and bone resorption around total hip stems and the effects of flexible materials. Clin Orthop 1992;274:124-34.

28. Van Rietbergen B, Huiskes R, Weinans $\mathbf{H}$, et al. The mechanism of bone remodeling and resorption around press-fitted THA stems. $J$ Biomech 1993;26:369-82.

29. Weinans H, Huiskes R, Van Rietbergen B, et al. Adaptive bone remodeling around bonded non-cemented total hip arthroplasty: a comparison between animal experiments and computer simulation. $J$ Orthop Res 1993;11:500-13.

30. Weinans H, Huiskes R, Verdenschot $N$, Van Rietbergen B. The effect of adaptive bone remodeling threshold levels on resorption around noncemented hip stems. In: Vanderby R, Jr, Ed. 1991 Advances in bioengineering. New York: American Society of Mechanical Engineers, 1991:303-6.

31. Huiskes R. Bone remodeling around implants can be explained as an effect of mechanical adaptations. In: Galante JO, Rosenberg AG, Gallaghan JJ, eds. Total hip revision surgery. New York: Raven Press Ltd, 1995:159-71.

32. Cadambi A, Engh GA, Dwyer KA, Vinh TN. Osteolysis of the distal femur after total knee arthroplasty. J Arthroplasty 1994;9:579-94.

33. Sychterz CJ, Engh CA. The influence of clinical factors on periprosthetic bone remodeling. Clin Orthop 1996;322:285-92.

34. Robertson DD, Mintzer CM, Weissman BN, et al. Distal loss of femoral bone following total knee arthroplasty. Measurement with visual and computer-processing of roentgenograms and dual-energy X-ray absorptiometry. J Bone Joint Surg [Am] 1994;76-A:66-76.

35. Weinans H, Huiskes R, Grootenboer HJ. Effects of fit and bonding characteristics of femoral stems on adaptive bone remodeling. $J$ Biomech Eng 1994;116:393-400.

36. Huiskes R, Van Rietbergen B. Preclinical testing of total hip stems: the effects of coating placement. Clin Orthop 1995;319:64-76. 